\title{
Compuestos de matriz metálica reforzada con carburos cementados para aplicaciones en herramientas agroindustriales
}

\section{Metal matrix composites reinforced with cemented carbides for applications in agricultural tools}

Presentación: 06/10/2020

\section{Doctorando:}

\section{Carolina García Díaz}

Grupo Tecnología de Procesos, Dpto. Metalurgia, Facultad Regional San Nicolás, Universidad Tecnológica Nacional / Centro de Investigación y Transferencia de San Nicolás (Consejo Nacional de Investigaciones Científicas y Técnicas) - Argentina cgarciadiaz@frsn.utn.edu.ar

\section{Director:}

\section{José Luis García}

\section{Co-director:}

\section{Edgardo Roque Benavidez}

\section{Resumen}

Actualmente, los recubrimientos de compuestos de matriz metálica reforzada aplicados sobre una base o sustrato de acero se utilizan en diferentes herramientas agrícolas. Sin embargo, estos materiales no se fabrican en el país, lo que conlleva un manejo elevado de costos y la imposibilidad de reaplicar el recubrimiento cuando se desgasta. En el presente trabajo se analiza una cuchilla comercial importada que posee un recubrimiento de matriz metálica reforzada con carburo de tungsteno. Mediante el análisis microestructural, análisis semicuantitativo EDS y perfil de microdureza, se determinaron las características de referencia para el diseño de recubrimientos propios. Los resultados del presente estudio permitieron inferir el método de aplicación y consolidación del recubrimiento y definir un punto de partida para la tesis en cuestión.

Palabras clave: Compuestos de matriz metálica reforzada, recubrimientos, herramientas agrícolas

\begin{abstract}
Currently, reinforced metal matrix composite coatings applied onto steel substrate are used in different agricultural tools. However, these materials are not manufactured in the country, which leads to high cost management and the inability to reapply the coating when it wears out. In the present work an imported commercial blade that has a metal matrix coating reinforced with tungsten carbide is analysed. Through microstructural analysis, semi-quantitative analysis EDS and microhardness profile, the reference characteristics for the design of own coatings were determined. The results of the present study allowed to infer the method of application and consolidation of the coating and to define a starting point for the thesis in question.
\end{abstract}

Keywords: Reinforced metal matrix composites, coatings, agricultural tools 


\section{Introducción}

Los materiales compuestos de alta resistencia al desgaste (compuestos por fases duras refractarias y matrices metálicas) cuentan con escaso nivel de investigación y desarrollo en nuestro país, considerándose un área de desarrollo vacante con un gran potencial para aplicaciones industriales tanto a nivel regional (norte de Buenos Aires y sur de Santa $\mathrm{Fe}$ ) como nacional. Los materiales compuestos evolucionan a partir de la idea de combinar dos materiales básicamente diferentes con propiedades físicas y mecánicas disímiles para llegar a un producto cuyas propiedades finales son superiores a las de los componentes individuales. Los compuestos de matriz metálica reforzada con partículas (PRMMC, en inglés) han encontrado aplicaciones en muchas áreas, especialmente en alta temperatura (Otsubo et al., 1999). El refuerzo con materiales cerámicos da como resultado un aumento en la resistencia, rigidez, resistencia al desgaste, resistencia a altas temperaturas y una disminución en el peso (Simunovic et al., 2014) (Ortner et al., 2014) (Brookes, 1996). En particular, la zona donde se desarrollará la tesis doctoral (San Nicolás, Prov. Bs.As.) es una zona agrícolaganadera por excelencia y es el sustento de un gran número de industrias dedicadas al agro. Entre los materiales que se utilizan para la producción de herramientas de maquinarias agrícolas (discos de sembradora, barras de trilla, cuchillas para picadora de forraje, etc.), el más destacado es el acero al boro. El mismo se utiliza generalmente en estado de templado y revenido, y en algunos casos muy puntuales donde la aplicación lo amerita, se le aplica un recubrimiento de compuestos de matriz metálica reforzada (Otsubo et al., 1999). En Argentina hay fabricación local de herramientas para el agro de acero al boro SAE 15B30 de producción nacional pero en materia de aplicación de recubrimientos duros, la producción es muy escasa y generalmente no se aplica a la industria del agro debido a que los costos son muy elevados. Por lo tanto, las herramientas que poseen este tipo de recubrimientos resistentes al desgaste son importadas. En particular, el objetivo de la tesis es mejorar el rendimiento y la durabilidad de herramientas utilizadas en la industria del agro mediante la aplicación de recubrimientos de compuestos de matriz metálica reforzada sobre un acero base SAE 15B30. Estos recubrimientos serán depositados y consolidados por diferentes técnicas para evaluar la adhesión de cada una de ellas. Mediante el análisis de interfaces, evaluación de la microestructura y ensayos físicos y mecánicos, se pretende elegir la mejor opción "material de recubrimiento + técnica de aplicación". El objetivo es dar una solución rentable y eficaz a los usuarios de estas herramientas, ya que hoy en día no está desarrollada en el país la potencialidad de recubrimientos en esta área tan importante para la economía. Como material de referencia, en este trabajo, se analiza una cuchilla de picadora de forraje comercial importada para evaluar las características del recubrimiento. De esta forma, en una siguiente etapa, se puede diseñar el recubrimiento propio, considerando que la herramienta comercial tiene amplia aceptación en el mercado local.

\section{Desarrollo}

Se seleccionó una cuchilla de picadora de forraje con recubrimiento anti-desgaste. Este tipo de herramientas se comercializan bajo el título de tener un recubrimiento de carburo de tungsteno (Ortner et al., 2014) (Brookes, 1996) (García et al., 2019) de grano grande con altas propiedades adhesivas, que asegura una mayor resistencia al desgaste y por lo tanto mayor durabilidad de las piezas. En la figura 1 se puede observar la pieza en cuestión. El cuerpo es de acero al boro y la zona del filo está templada por inducción (Figura 1a), según informa el fabricante. El recubrimiento de carburo de tungsteno se aprecia en la parte posterior del filo (Figura 1b). 

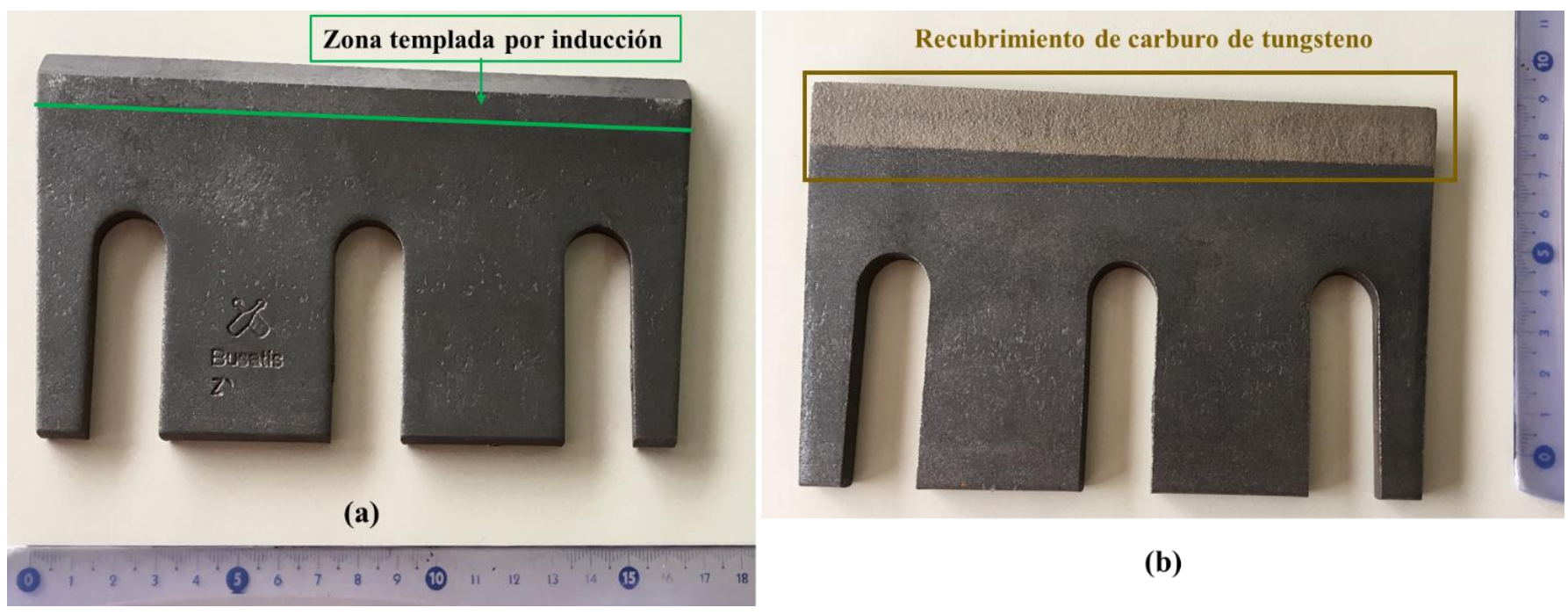

(b)

Figura 1: Cuchilla de heno estándar con recubrimiento de carburo de tungsteno, (a) vista anterior donde se aprecia el filo templado por inducción, (b) vista posterior donde se aprecia el recubrimiento anti-desgaste.

Las muestras para el análisis microestructural se prepararon cortando secciones longitudinales correspondientes al acero base, la interfase y al filo, seguido de pulido grueso (con papeles de SiC) y fino con pasta de diamante de hasta $3 \mu \mathrm{m}$. Para una mejor observación de la estructura, las muestras se atacaron con Nital 2. La determinación de las fases y el tamaño de grano en la zona del filo y del material base (acero al boro) se obtuvieron mediante observaciones en un microscopio óptico Olympus GX51 y por microscopía electrónica de barrido (FE-SEM), utilizando un microscopio Sigma Zeiss equipado con una pistola de emisión de campo tipo Schottky y con detectores de electrones secundarios y retrodifundidos. Los perfiles de microdureza Vickers (HV0.3) se realizaron en la sección transversal de muestras pulidas con un microdurómetro Leco LM-300AT.

La microestructura del acero base está constituida en su totalidad por martensita (figura 2a), ya que este tipo de acero se utiliza en piezas para maquinarias y herramientas agrícolas en estado de templado y revenido para aumentar su resistencia al desgaste. En la interfase entre el acero base y el acero del filo (tratado por inducción) se puede observar la diferencia en el tamaño de las agujas de martensita (figura 2b), siendo más finas las del acero templado por inducción (zona del filo de la cuchilla). En la figura 2c se muestra un detalle del acero del filo. El recubrimiento (figura 2d) está compuesto por granos de carburo de tungsteno (gris claro) de morfología nodular embebidos en una matriz (gris oscuro). El tamaño observado de los granos de carburo de tungsteno (WC) se encuentra mayoritariamente entre 20 y $70 \mu \mathrm{m}$. El espesor del recubrimiento varía entre 95 y $225 \mu \mathrm{m}$. 


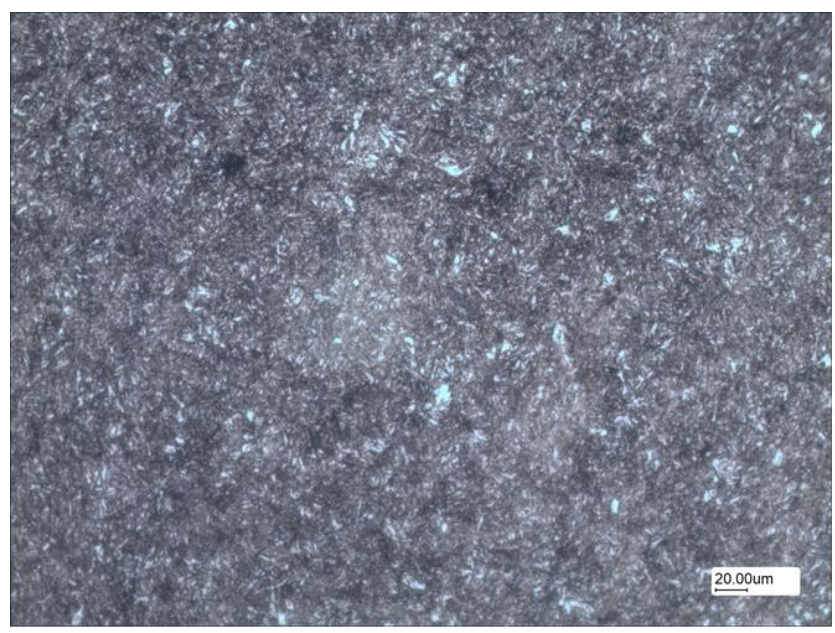

(a)

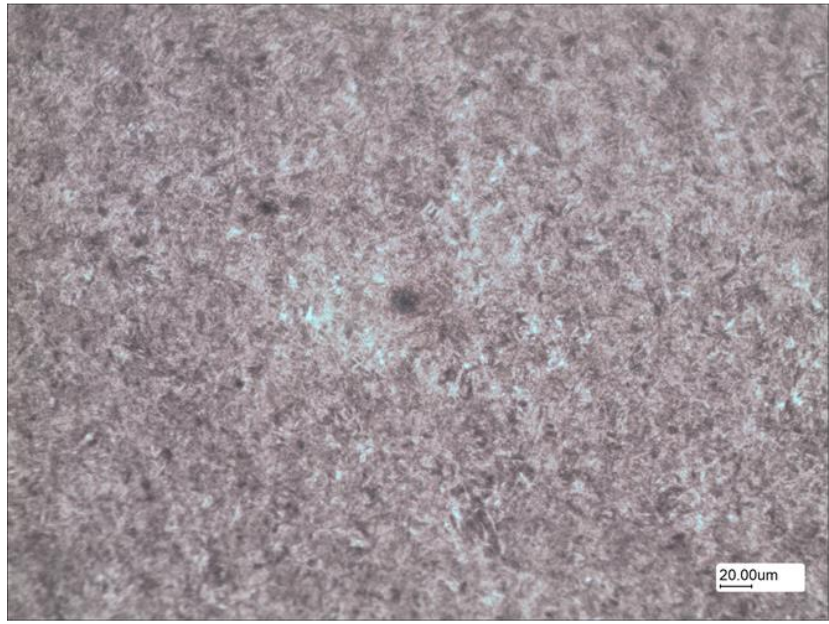

(c)

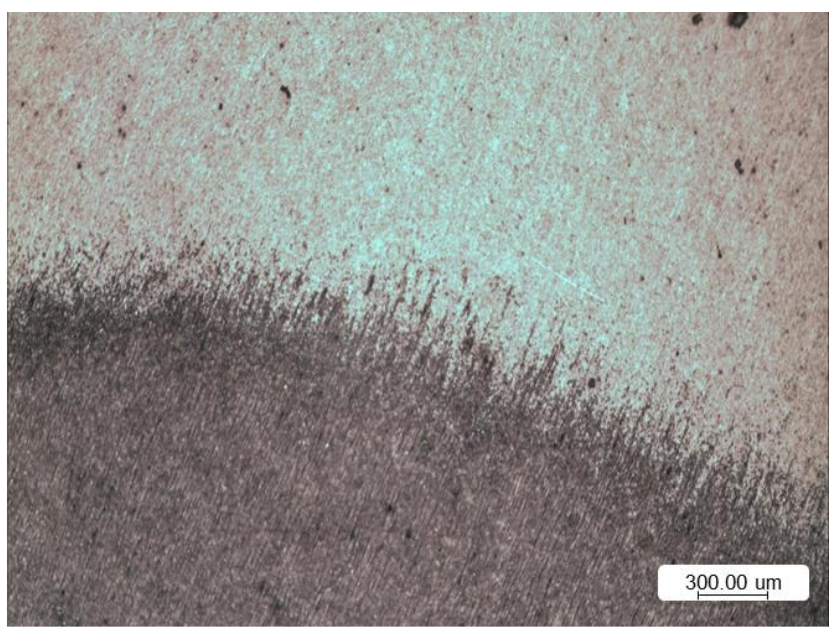

(b)

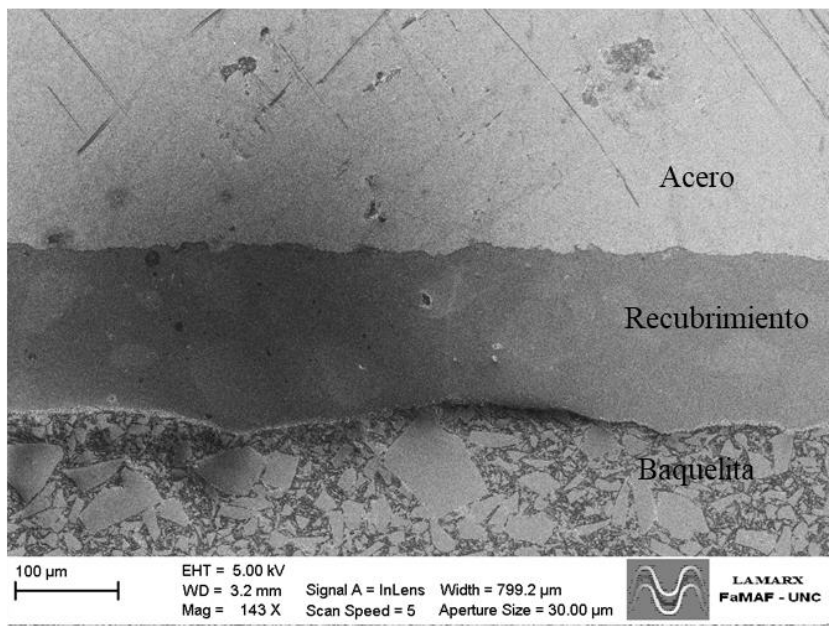

(d)

Figura 2: Microscopía óptica de (a) acero base a 500x; (b) interfase de acero base y acero del filo a 50x; y (c) acero del filo templado por inducción a 500x. (d) Microscopía electrónica de barrido del recubrimiento de carburo de tungsteno.

El análisis semicuantitativo EDS muestra los posibles elementos que conforman el recubrimiento. En la figura 3 se observa que los granos grandes son efectivamente de carburo de tungsteno. La matriz metálica del recubrimiento está compuesta principalmente por níquel, cromo, hierro y, en menor proporción, cobalto. Con estos resultados, podemos estimar que estamos frente a un compuesto de matriz metálica reforzada con carburo de tungsteno (Iždinská et al., 2010). La matriz metálica en este tipo de materiales es en general una superaleación de base níquel o, en algunos casos, de base hierro (Simunovic et al., 2014) (Lee et al., 2017). En el caso particular de esta cuchilla comercial se puede deducir que la matriz metálica se trata de una superaleación de base níquel (Simunovic et al., 2014) (Iždinská et al., 2010). Teniendo en cuenta que el espesor del recubrimiento no es homogéneo, el tipo de morfología de los granos de carburo de tungsteno y sabiendo que el acero del filo está tratado por inducción, se puede inferir que este material se deposita por un método químico (Benavidez, 2016) y se consolida al realizar el temple del acero en esa zona. 
W M 1

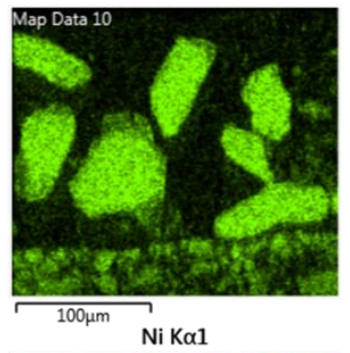

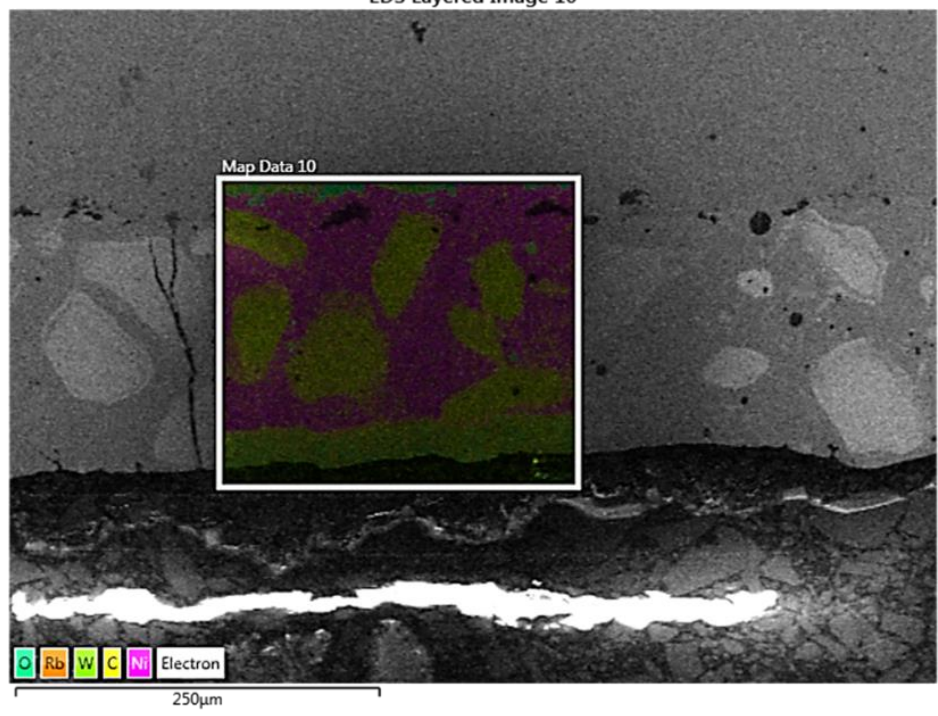

EDS Layered Image 10
$\mathrm{C} K \alpha 12$

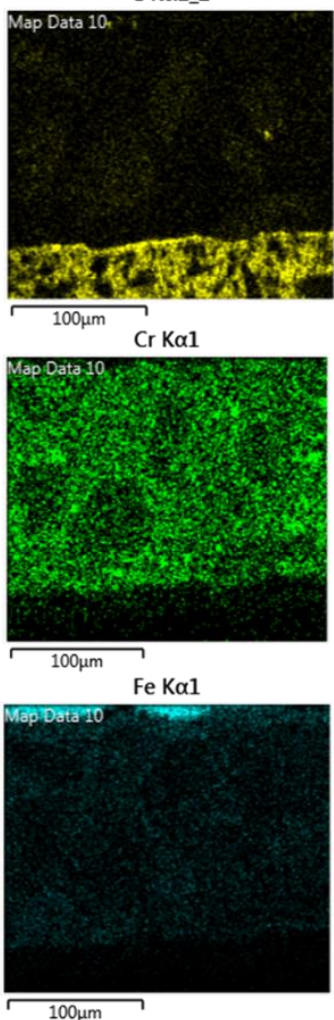

Figura 3: Análisis de EDS de posibles elementos que integran el recubrimiento de la herramienta.

Se realizaron un promedio de 13 mediciones de microdureza en el acero base, en el acero del filo y en el recubrimiento con una carga de $0,3 \mathrm{~kg}$. Las mediciones de microdureza en el recubrimiento se realizaron en la matriz metálica y sobre los granos de carburo de tungsteno por separado.

La microdureza promedio del material base de acero es de $400 \pm 15 \mathrm{HV} 0.3$, mientras que la del acero del filo es de 610 $\pm 25 \mathrm{HV}$ 0.3. La microdureza promedio del carburo de tungsteno es $1590 \pm 300 \mathrm{HV} 0.3$. Es posible que el WC utilizado sea producido mediante fusión y trituración ya que la microdureza es menor que el producto fabricado mediante sinterización (usualmente del orden de 2000-2500 HV0.3) (García et al., 2019). Además el tamaño de partícula es mucho mayor al tamaño obtenido por métodos pulvimetalúrgicos (menor a $5 \mu \mathrm{m}$ ) (García et al., 2019). Por otro lado, la microdureza promedio de la matriz metálica es de $630 \pm 180 \mathrm{HV0.3}$. La variabilidad de los valores de microdureza medidos en el recubrimiento probablemente se deban a la heterogeneidad de las fases presentes: granos de carburo de tungsteno y la matriz metálica ( $\mathrm{Ni}, \mathrm{Cr}, \mathrm{Fe}, \mathrm{Co}$, etc.). En la propia matriz metálica se pueden formar fases (por ejemplo, $\mathrm{Cr}_{2} \mathrm{C}_{3}$ ) más duras que otras durante la consolidación del recubrimiento, mientras que en los granos de WC la microdureza puede variar dependiendo la zona en las que se tomaron las mediciones, es decir, en el centro del grano o más cerca al límite del grano. En la figura 4 se puede apreciar la comparación de los valores de microdureza de las distintas fases. Las mediciones de microdureza se correlacionan con el análisis microestructural. Un punto a destacar es que la microdureza del acero del filo presenta un valor (promedio) similar al de la matriz metálica del recubrimiento. 


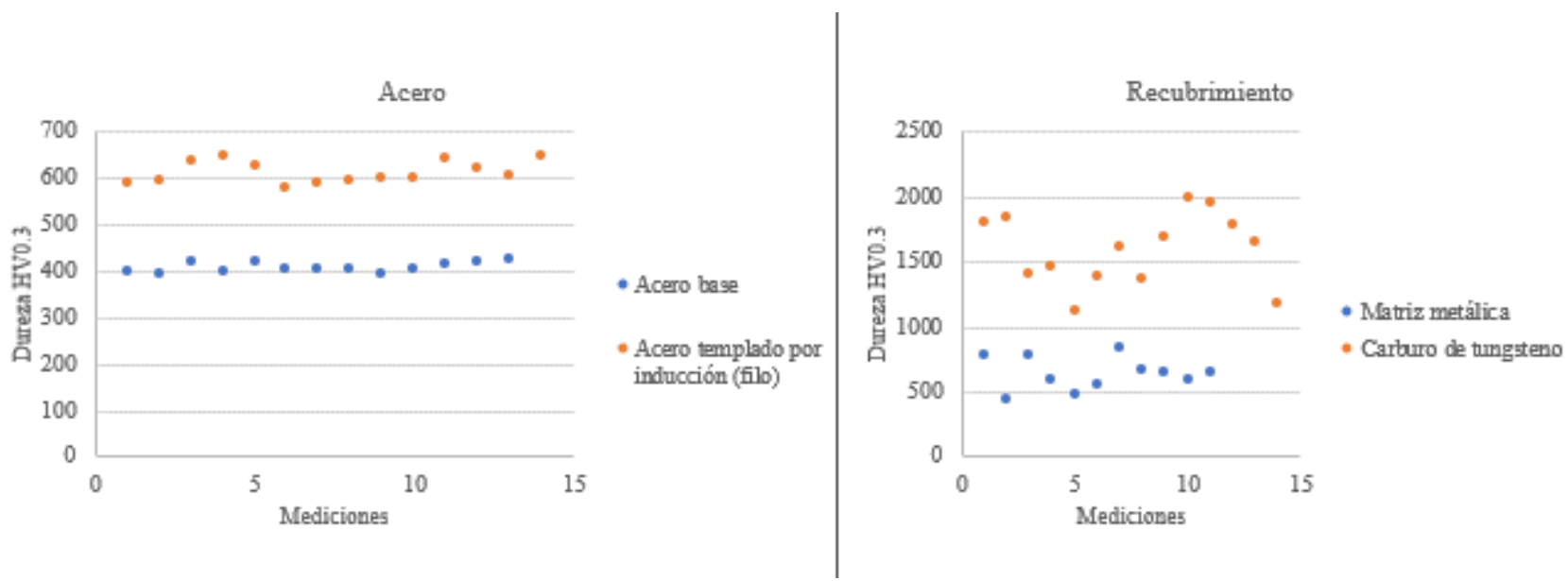

Figura 4: Perfiles de microdureza del acero base y del filo y del recubrimiento.

\section{Conclusiones}

El presente estudio permitió conocer las características microestructurales, la microdureza y los principales componentes del material de referencia. Estos resultados permitieron inferir el método de aplicación del recubrimiento, lo que nos permite sentar las bases para diseñar nuestra propia aleación metálica y refuerzo de carburo y, además, nuestra propia técnica de deposición y consolidación. Para ello se deberán realizar simulaciones termodinámicas para obtener composiciones óptimas y las temperaturas de fusión de las aleaciones para aplicar al acero base. De esta forma se busca obtener un recubrimiento PRMMC con un balance de dureza y tenacidad adecuado, así como una buena adhesión al acero base, evitando la presencia de fases indeseables como sub-carburos frágiles. Luego, se deberá aplicar el recubrimiento mediante alguna técnica de deposición física o química y consolidarlo con el método más adecuado y económicamente rentable. No se descarta realizar un tratamiento térmico o mecánico para homogenizar la microestructura, relajar esfuerzos residuales y optimizar el componente acero/recubrimiento luego del proceso de deposición.

\section{Referencias}

Benavidez, E. R. (2016). Comportamiento a altas temperaturas del sistema YBa2Cu307-x + Ag. Saarbrücken, Alemania: Editorial Académica Española.

Brookes, K. J. A. (1996). World Directory and Handbook of Hardmetals and Hard Materials. Recuperado de https://books.google.com.ar/

Iždinská, Z., Nasher, A., y Iždinský, K. (2010). The structure and properties of composite laser clad coatings with Ni-based matrix with WC particles. Mater. Engineering, 17(2).

Lee, Y. T. R., Ashrafizadeh, H., Fisher, G., y McDonald, A. (2017). Effect of type of reinforcing particles on the deposition efficiency and wear resistance of low-pressure cold-sprayed metal matrix composite coatings. Surf. \& Coatings Tech, 324, 190-200.

García, J., Collado Ciprés, V., Kaplan, B., y Blomqvist, A. (2019). Cemented carbide microstructures: a review. Int. J. Refract. Met. Hard Mater, 80, 40-68.

Ortner, H. M., Kolaska, H., y Ettmayer, P. (2014). The history of the technological progress of hardmetals. Int. J. Refract. Met. Hard Mater, 44, 148-159.

Otsubo, F., Era, H., y Kishitake, K. (1999). Interface Reaction between Nickel-Base Self-Fluxing Alloy Coating and Steel Substrate. J. Therm. Spray Tech, 9, 259.

Simunovic, K., Saric, T., y Simunovic, G. (2014). Different Approaches to the Investigation and Testing of the Ni-Based SelfFluxing Alloy Coatings-A Review. Part 1: General Facts, Wear and Corrosion Investigations. Tribology Transactions, 57(6), 955-979. 